\title{
Primary intrahepatic malignant mesothelioma with multiple lymphadenopathies due to non-tuberculous mycobacteria: A case report and review of the literature
}

\author{
NORIKO INAGAKI, KAYOKO KIBATA, TAKESHI TAMAKI, TOSHIKI SHIMIZU and SHOSAKU NOMURA
}

First Department of Internal Medicine, Kansai Medical University, Moriguchi, Osaka 570-8506, Japan

Received March 28, 2013; Accepted July 9, 2013

DOI: 10.3892/ol.2013.1461

\begin{abstract}
Primary intrahepatic malignant mesothelioma (PIHMM) is an extremely rare tumor with clinicopathological characteristics that remain to be elucidated. The current study presents the case of a 68-year-old female with PIHMM and multiple lymphadenopathies due to non-tuberculous mycobacteria. The patient presented with an intrahepatic tumor, $70 \mathrm{~mm}$ in diameter, in the right lobe of the liver. An ultrasound-guided fine-needle aspiration biopsy of the liver tumor revealed findings that were consistent with an intrahepatic malignant mesothelioma. The systemic lymph node swellings were due to epithelioid granulomas that were caused by non-tuberculous mycobacteria. However, a hepatic rupture occurred due to the rapid growth of the liver tumor and consequently, a surgical resection was not performed. A review of the literature revealed that the clinicopathological characteristics of PIHMM are similar to those of non-occupational mesothelioma. However, PIHMM is usually a solitary tumor and is rarely associated with cavity effusion in contrast with conventional mesothelioma. Therefore, surgical resection with curative intent is often recommended for patients with PIHMM.
\end{abstract}

\section{Introduction}

Malignant mesothelioma most commonly arises from the pleura (1), but it may also arise from the peritoneum (2), pericardium (3) and tunica vaginalis testis $(4,5)$. However, primary intrahepatic malignant mesothelioma (PIHMM) is an extremely rare tumor (6-11). Malignant mesothelioma is known to originate from transformed mesothelial cells (12), which are not present in the hepatic parenchyma under normal physiological conditions. A possible explanation for the origin of PIHMM was proposed by Leonardou et al (7), who

Correspondence to: Dr Toshiki Shimizu, First Department of Internal Medicine, Kansai Medical University, Fumizono-cho 10-15, Morihuchi, Osaka 570-8506, Japan

E-mail: shimizto@takii.kmu.ac.jp

Key words: malignant mesothelioma, primary hepatic tumor, mycobacteria, literature review speculated that the tumor arose from mesothelial cells derived from an intruded Glisson's capsule. However, the clinicopathological characteristics of PIHMM remain to be elucidated. The current study reports a case of PIHMM with multiple lymphadenopathies due to non-tuberculous mycobacteria and also presents the findings of a literature review. Written informed consent was obtained from the patient.

\section{Case report}

A 68-year-old female presented to Kansai Medical University Takii Hospital (Osaka, Japan) with an intrahepatic tumor and multiple lymph node swellings accompanied by a prolonged low-grade fever. The patient did not have a history of asbestos exposure or cigarette smoking. A computed tomography (CT) scan revealed cervical, axillary and abdominal para-aortic lymph node swellings, in addition to an intrahepatic tumor with a diameter of $70 \mathrm{~mm}$ in the right lobe of the liver. The intrahepatic tumor was heterogeneously enhanced by contrast-enhanced CT. There was no evidence of pleural effusion, ascites, pleural thickening or a peritoneal tumor. Subsequently, 2-deoxy-2 $\left[{ }^{18} \mathrm{~F}\right]$-fluoro-D-glucose (FDG)-positron emission tomography(PET)/CT was performed, which clearly revealed a high FDG uptake in the lymph nodes and intrahepatic tumor (Fig. 1A-D). In contrast, no significant accumulation of FDG was noted in the pleura or the peritoneum. A laboratory examination showed that the C-reactive protein (CRP) and lactic dehydrogenase (LDH) levels were slightly elevated (CRP, $3.907 \mathrm{mg} / \mathrm{dl}$; LDH, $247 \mathrm{U} / \mathrm{l}$ ). However, the serum tumor marker levels were not elevated. The results of all the other laboratory examinations were within normal limits.

An ultrasound-guided fine-needle aspiration biopsy of the liver tumor was performed. A histological examination of the biopsy specimen revealed islands of polygonal tumor cells with a high nuclear to cytoplasmic ratio and prominent nucleoli (Fig. 2A). Few mitotic figures were noted. The tumor islands were surrounded by inflammatory infiltrates. Alcian blue and periodic acid-Schiff staining clearly demonstrated intracytoplasmic mucopolysaccharides in the tumor cells.

Immunohistochemical examination revealed that the tumor cells were negative for carcinoembryonic antigen, carbohydrate antigen 19-9, p53 and CD34. In contrast, the tumor cells stained positive for epithelial membrane protein, cytokeratin (CK) 7, CK20, CD10 and vimentin. In addition, 
A



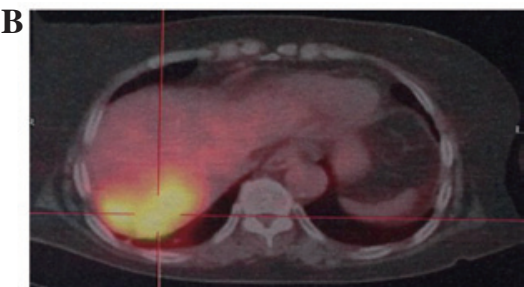

C

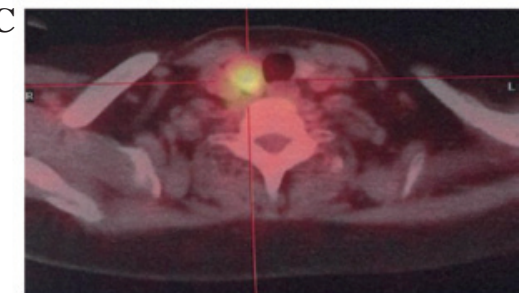

D

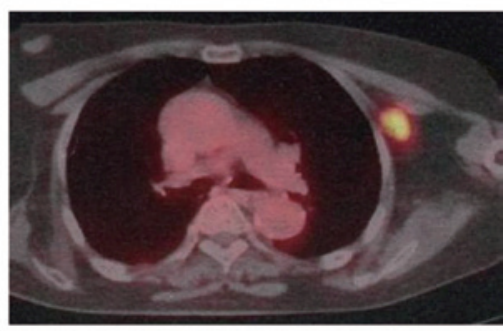

Figure 1. Radiological findings. (A) The fusion images of FDG-PET/CT in the coronal plane. (B) A lesion exhibiting a high FDG uptake (SUVmax, $>13.5$ ) was detected in the intrahepatic tumor of the right lobe of the liver. (C) The cervical lymph node demonstrated a high FDG uptake (SUVmax, $>10.6)$. (D) The left axillary gland exhibited a high FDG uptake (SUVmax, >8.4). FDG-PET/CT, 2-deoxy-2-[ $\left.{ }^{18} \mathrm{~F}\right]$-fluoro-D-glucose positron emission tomography/computer tomography, SUVmax, maximum standard uptake value.
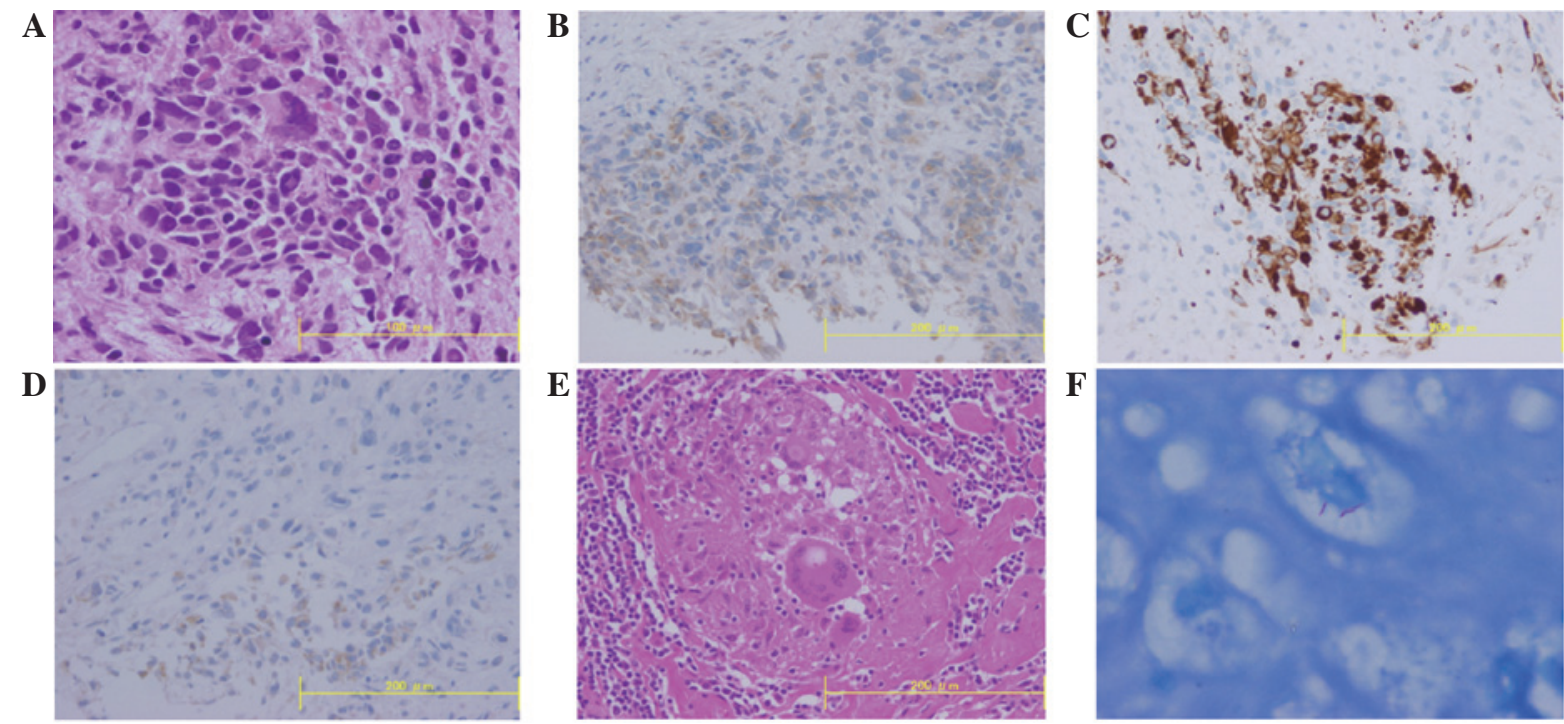

Figure 2. Histological findings of the biopsy specimen. (A-D) The histological findings of the biopsy specimen from the intrahepatic tumor. (A) Results of HE staining revealed the islands of polygonal tumor cells surrounded by inflammatory infiltrates (magnification, $x 400$ ). (B-D) Immunohistochemical findings of the biopsy specimen from the intrahepatic tumor. (B) The tumor cells were immunoreactive for cytoplasmic calretinin (magnification, x200). (C) The tumor cells stained positive for Wilms tumor gene-1 (WT-1; magnification, x200). (D) The tumor cells were immunoreactive for cytoplasmic D2-40 (magnification, x200). (E and F) Histological findings of the biopsy specimen from the left axillary lymph node. (E) An epithelioid granuloma with multinucleated giant cells was observed in the lymph node (HE staining; magnification, x200). (F) Ziehl-Neelsen acid-fast staining showed the presence of acid-fast bacilli (x1,000). HE, hematoxylin and eosin.

immunohistochemical staining revealed that the tumor cells were positive for calretinin, Wilms tumor gene-1 (WT-1) and D2-40 (Fig. 2B-D). These findings strongly suggested that the intrahepatic tumor cells exhibited the phenotypical features of malignant mesothelioma.

An axillary lymph node biopsy was performed to determine whether the lymph node swellings were due to intrahepatic mesothelioma metastasis. However, the histological examination showed an epithelioid granuloma (Fig. 2E) and Ziehl-Neelsen staining revealed the presence of acid-fast bacilli (Fig. 2F). Thus, a final diagnosis of PIHMM accompanied by lymphadenopathies due to a mycobacterial infection was confirmed. PCR for Mycobacterium tuberculosis and the avium-intracellulare complex was negative. The cultiva- 
Table I. Characteristics of patients with PIHMM.

\begin{tabular}{|c|c|c|c|c|c|c|c|c|c|}
\hline First author, year (ref.) & $\begin{array}{l}\text { Age, } \\
\text { years }\end{array}$ & Gender & $\begin{array}{l}\text { Asbestos } \\
\text { exposure }\end{array}$ & Histology & $\begin{array}{c}\text { OS, } \\
\text { months }\end{array}$ & $\begin{array}{l}\text { Location } \\
\text { (segment) }\end{array}$ & $\begin{array}{l}\text { Size, } \\
\mathrm{cm}\end{array}$ & Treatment & Relapse \\
\hline Imura et al, 2002 (6) & 64 & M & $(-)$ & Ep & 40 & Rt (S7) & 3.2 & Surg & None \\
\hline Leonardou et al, 2003 (7) & 54 & $\mathrm{~F}$ & $\mathrm{~N} / \mathrm{E}$ & Ep & 2 & Rt & 16.0 & Surg & None \\
\hline Gütgement et al, 2006 (8) & 62 & M & $(-)$ & Ep & 5 & Rt & 5.8 & Surg & LNR \\
\hline Kim et al, 2008 (9) & 53 & M & $(-)$ & $\mathrm{Bp}$ & $\mathrm{N} / \mathrm{E}$ & Rt & 13.0 & Surg & DI \\
\hline Sasaki et al, 2009 (10) & 66 & M & $(+)$ & $\mathrm{Bp}$ & 6 & Rt (S8) & 4.4 & Surg & None \\
\hline Buchholz et al, 2009 (11) & 62 & M & $(-)$ & Ep & 36 & Rt $(\mathrm{S} 5, \mathrm{~S} 8)$ & 5.8 & Surg & LNR \\
\hline Present case & 68 & $\mathrm{~F}$ & $(-)$ & Ep & 3 & Rt (S7) & 7.0 & BSC & $\mathrm{N} / \mathrm{E}$ \\
\hline
\end{tabular}

PIHMM, primary intrahepatic malignant mesothelioma; OS, overall survival time; M, male; F, female; N/E, not evaluated; Ep, epithelioid; Bp, biphasic; Surg, surgical resection; BSC, best supportive care; Rt, right lobe; LNR, translymphatic relapse; DI, direct invasion.

Table II. Immunohistochemical phenotypes of PIHMM and conventional mesothelioma.

\begin{tabular}{|c|c|c|c|c|c|c|c|}
\hline Items & D2-40 & WT-1 & Calretinin & Vimentin & p53 & CK7 & CK20 \\
\hline \multicolumn{8}{|l|}{ PIHMM, degree of staining } \\
\hline Imura et al, 2002 (6) & $\mathrm{N} / \mathrm{E}$ & $\mathrm{N} / \mathrm{E}$ & $(+)$ & $\mathrm{N} / \mathrm{E}$ & $(+)$ & $\mathrm{N} / \mathrm{E}$ & $\mathrm{N} / \mathrm{E}$ \\
\hline Leonardou et al, 2003 (7) & $\mathrm{N} / \mathrm{E}$ & $\mathrm{N} / \mathrm{E}$ & $(+)$ & $(+)$ & $\mathrm{N} / \mathrm{E}$ & $\mathrm{N} / \mathrm{E}$ & $\mathrm{N} / \mathrm{E}$ \\
\hline Gütgement et al, 2006 (8) & $(+)$ & $(+)$ & $(+)$ & $( \pm)$ & $(+)$ & $\mathrm{N} / \mathrm{E}$ & $(-)$ \\
\hline Kim et al, 2008 (9) & $\mathrm{N} / \mathrm{E}$ & $\mathrm{N} / \mathrm{E}$ & $(+)$ & $\mathrm{N} / \mathrm{E}$ & $\mathrm{N} / \mathrm{E}$ & $(+)$ & $(-)$ \\
\hline Sasaki et al, 2009 (10) & $(+)$ & $(+)$ & $(+)$ & $(+)$ & $(+)$ & $(+)$ & N/E \\
\hline Buchholz et al, 2009 (11) & $(+)$ & $(+)$ & $(+)$ & $( \pm)$ & $(+)$ & $\mathrm{N} / \mathrm{E}$ & $(-)$ \\
\hline Present case & $( \pm)$ & $(+)$ & $(+)$ & $(+)$ & $(-)$ & $(+)$ & $(+)$ \\
\hline $\begin{array}{l}\text { Conventional mesothelioma }{ }^{\mathrm{a}}, \% \\
\text { (refs.) }\end{array}$ & $\begin{array}{l}85 \\
(20)\end{array}$ & $\begin{array}{c}55-100 \\
(20,21,23)\end{array}$ & $\begin{array}{c}39.8-100 \\
(20-22)\end{array}$ & $\begin{array}{c}27.7-96 \\
(21,22,24)\end{array}$ & $\begin{array}{l}45-69.6 \\
(22,25)\end{array}$ & $\begin{array}{l}65-100 \\
(26-28)\end{array}$ & $\begin{array}{c}0 \\
(26-28)\end{array}$ \\
\hline
\end{tabular}

${ }^{a}$ The proportion of positive staining (refs. 20-28). PIHMM, primary intrahepatic malignant mesothelioma; N/E, not evaluated; WT-1, Wilms tumor gene-1; CK, cytokeratin; (+), positive staining; (-), negative staining; ( \pm ), weakly positive staining.

tion for acid-fast bacillus failed to yield a mycobacterium species. Therefore, the pathogenic mycobacterium was not determined in this case. Since the lymphadenopathy was not due to mesothelioma metastasis, the PIHMM was considered to be a localized tumor. Therefore, a surgical resection of the liver tumor with curative intent was planned. However, a hepatic rupture occurred due to the rapid growth of the liver tumor and the general condition of the patient deteriorated. Therefore, no further investigations or treatments were possible in this case.

\section{Discussion}

PIHMM is an extremely rare tumor. To the best of our knowledge, only six cases of PIHMM have been previously reported in the published literature (6-11). A review of these six cases, plus the present study, is summarized in Table I. The cases consisted of five male and two female patients (2.5:1), with an age range of 53-68 years (median, 62 years). Previous reviews focusing on conventional mesothelioma have shown that the male/female ratio and median age at the initial diagnosis ranged from 2.2:1-12.6:1 and 64-68 years, respectively (13-15).
However, in a subgroup of non-occupational mesothelioma cases, the male/female ratio and median age at the initial diagnosis were reported to be 0.8:1-1.4:1 and 57.8-63.0 years, respectively $(13,16)$. Thus, gender and age distribution did not differ significantly between PIHMM and non-occupational mesothelioma. Only one of the seven patients (14.3\%) had a history of asbestos exposure, although it has previously been shown that conventional mesothelioma is frequently associated with asbestos exposure (58.9-86.8\%) (13-15).

The prevalence of distant metastasis at the initial diagnosis has been recorded as $25.2-55.1 \%$ in conventional mesothelioma $(13,16)$. Therefore, surgical resection is not always performed. However, all seven of the PIHMM patients reviewed in the present study had solitary tumors that were localized in the liver at the time of the initial diagnosis, and surgical resection had been performed in all cases, with the exception of the present case. All the tumors arose in the right lobe, were located in the subcapsular region and were between 3.2 and $16 \mathrm{~cm}$ in diameter (mean, $7.8 \mathrm{~cm}$ ). Cavity effusion was not associated with PIHMM in any of the reviewed cases, however malignant serositis is usually observed in conventional mesothelioma (13). Three of the six patients that underwent a 
surgical resection relapsed post-surgery, one of which received systemic chemotherapy with pemetrexed in combination with cisplatin. Two of the three relapsed cases showed translymphatic progression. The prevalence of lymph node metastasis in conventional mesothelioma has been evaluated. Rahman et al reported that 18 of 53 patients $(34.0 \%)$ with malignant pleural mesothelioma had positive lymph node involvement at the time of surgery (17). In addition, Edwards et al reported that 44 of 92 consecutive patients $(47.8 \%)$ with malignant mesothelioma who underwent extrapleural pneumonectomy had positive lymph node involvement (18). Therefore, translymphatic progression is not an unusual event in malignant mesothelioma. In the present study, the survival factors were not evaluated due to the small size of the study population and inadequate survival information.

The review of the seven cases showed that five were epithelioid type (71.4\%) and two were biphasic (28.6\%). A sarcomatoid type was not noted among the reviewed cases. Among the conventional mesothelioma cases, the prevalence of epithelioid, biphasic and sarcomatoid subtypes was 32-67.2, 21.7-34 and 9.8-33\%, respectively (13-16). The distribution of the histological types among the non-occupational mesotheliomas was not dissimilar to that among the conventional mesotheliomas (epithelioid, 64.9\%; biphasic, $15.3 \%$ and sarcomatoid, 6.1\%) (19). Therefore, the distribution of the histological subtypes in PIHMM was equivalent to that in conventional mesothelioma. The tumor phenotypes of the reviewed cases are summarized in Table II. Previously, several studies had been conducted to clarify the phenotypical features of malignant mesothelioma using immunohistochemistry and these results are also listed in Table II. Consequently, this literature review clearly showed that PIHMM and conventional mesothelioma do not differ significantly with respect to cellular phenotype (20-28).

In the present case, multiple lymphadenopathies were observed in addition to the liver tumor. FDG-PET/CT did not demonstrate the difference in metabolic behavior between the intrahepatic tumor and lymphadenopathies. However, the lymph node lesions were identified to be non-cancerous granulomas due to the presence of mycobacteria. FDG-PET/CT examination was insufficient to differentiate epithelioid granuloma due to mycobacterial infection from malignant mesothelioma in this case. Studies have also demonstrated that mycobacteriosis commonly causes increased ${ }^{18}$ F-FDG uptake (29-32). Thus, an aggressive biopsy is warranted when multiple lymph node swellings are associated with a malignant tumor.

The present study describes a case of PIHMM with multiple lymphadenopathies due to non-tuberculous mycobacteria. A literature review clearly indicated that the clinicopathological characteristics of PIHMM are similar to those of non-occupational mesothelioma. However, the tumors in all the reviewed cases were solitary tumors that were localized in the liver and none were accompanied by cavity effusion. Further investigation of the pathophysiological features of PIHMM is required to develop an appropriate treatment strategy.

\section{References}

1. Maitra A and Kumar V (eds): The lung and upper respiratory tract. Robbins Basic Pathology. 7th edition. Saunders, Philadelphia, pp453-509, 2002.
2. Battifora $\mathrm{H}$ and McCaughey E (eds): Tumors of the Serosal Membranes. Armed Forces Institute of Pathology, Washington, DC, 1994.

3. Thomason R, Schlegel W, Lucca M, et al: Primary malignant mesothelioma of the pericardium. Case report and literature review. Tex Heart Inst J 21: 170-174, 1994.

4. Kasdon EJ: Malignant mesothelioma of the tunica vaginalis propria testis. Report of two cases. Cancer 23: 1144-1150, 1969.

5. Kozlowski H and Zoltowska A: Mesothelioma of spermatic cord. Neoplasma 15: 97-100, 1968.

6. Imura J, Ichikawa K, Takeda J, Iwasaki Y, et al: Localized malignant mesothelioma of the epithelial type occurring as a primary hepatic neoplasm: a case report with review of the literature. APMIS 110: 789-794, 2002.

7. Leonardou P, Semelka RC, Kanematsu M, et al: Primary malignant mesothelioma of the liver: MR imaging findings. Magn Reson Imaging 21: 1091-1093, 2003.

8. Gütgemann I, Standop J and Fischer HP: Primary intrahepatic malignant mesothelioma of epithelioid type. Virchows Arch 448: 655-658, 2006.

9. Kim DS, Lee SG, Jun SY, et al: Primary malignant mesothelioma developed in liver. Hepatogastroenterology 55: 1081-1084, 2008.

10. Sasaki M, Araki I, Yasui T, et al: Primary localized malignant biphasic mesothelioma of the liver in a patient with asbestosis. World J Gastroenterol 15: 615-621, 2009.

11. Buchholz BM, Gütgemann I, Fischer HP, et al: Lymph node dissection in primary intrahepatic malignant mesothelioma: case report and implications for diagnosis and therapy. Langenbecks Arch Surg 394: 1123-1130, 2009.

12. Husain AN, Colby TV, Ordóñez NG, et al: Guidelines for pathologic diagnosis of malignant mesothelioma: a consensus statement from the International Mesothelioma Interest Group. Arch Pathol Lab Med 133: 1317-1331, 2009.

13. Yates DH, Corrin B, Stidolph PN and Browne K: Malignant mesothelioma in south east England: clinicopathological experience of 272 cases. Thorax 52: 507-512, 1997.

14. Borasio P, Berruti A, Billé A, et al: Malignant pleural mesothelioma: clinicopathologic and survival characteristics in a consecutive series of 394 patients. Eur J Cardiothorac Surg 33: 307-313, 2008.

15. Gemba K, Fujimoto N, Kato K, et al: National survey of malignant mesothelioma and asbestos exposure in Japan. Cancer Sci 103: 483-490, 2012.

16. Law MR, Hodson ME and Heard BE: Malignant mesothelioma of the pleura: relation between histological type and clinical behaviour. Thorax 37: 810-815, 1982.

17. Abdel Rahman AR, Gaafar RM, Baki HA, et al: Prevalence and pattern of lymph node metastasis in malignant pleural mesothelioma. Ann Thorac Surg 86: 391-395, 2008.

18. Edwards JG, Stewart DJ, Martin-Ucar A, et al: The pattern of lymph node involvement influences outcome after extrapleural pneumonectomy for malignant mesothelioma. J Thorac Cardiovasc Surg 131: 981-987, 2006.

19. Metintas M, Metintas S, Ak G, et al: Epidemiology of pleural mesothelioma in a population with non-occupational asbestos exposure. Respirology 13: 117-121, 2008.

20. Saad RS, Lindner JL, Lin X, et al: The diagnostic utility of D2-40 for malignant mesothelioma versus pulmonary carcinoma with pleural involvement. Diagn Cytopathol 34: 801-806, 2006.

21. Ordóñez NG: The immunohistochemical diagnosis of mesothelioma: A comparative of epithelioid mesothelioma and lung adenocarcinoma. Am J Surg Pathol 27: 1031-1051, 2003.

22. Roberts F, Harper CM, Downie I and Burnett RA: Immunohistochemical analysis still has a limited role in the diagnosis of malignant mesothelioma. A study of thirteen antibodies. Am J Clin Pathol 116: 253-262, 2001.

23. Pu RT, Pang Y and Michael CW: Utility of WT-1, p63, MOC31, mesothelin, and cytokeratin (K903 and CK5/6) immunostains in differentiating adenocarcinoma, squamous cell carcinoma, and malignant mesothelioma in effusions. Diagn Cytopathol 36: 20-25, 2008

24. Moch H, Oberholzer M, Christen H, et al: Diagnostic tools for differentiating pleural mesothelioma from lung adenocarcinoma in paraffin embedded tissue. II. Design of an expert system and its application to the diagnosis of mesothelioma. Virchows Arch A Pathol Anat Histopathol 423: 493-496, 1993.

25. Attanoos RL and Gibbs AR: Pathology of malignant mesothelioma. Histopathol 30: 403-418, 1997. 
26. Tot $\mathrm{T}$ : The value of cytokeratins 20 and 7 in discriminating metastatic adenocarcinoma from pleural mesotheliomas. Cancer 92: 2727-2732, 2001

27. Wang NP, Zee S, Zarbo RJ, et al: Coordinate expression of cytokeratins 7 and 20 defines unique subsets of carcinomas. Appl Immunohistochem 3: 99-107, 1995.

28. Chu P, Wu E and Weiss LM: Cytokeratin 7 and cytokeratin 20 expression in epithelial neoplasms: a survey of 435 cases. Mod Pathol 13: 962-972, 2000.

29. Treglia G, Taralli S, Calcagni ML, et al: Is there a role for fluorine 18 fluorodeoxyglucose-positron emission tomography and positron emission tomography/computed tomography in evaluating patients with mycobacteriosis? A systematic review. J Comput Assist Tomogr 35: 387-393, 2011.
30. Das CJ, Kumar R, Balakrishnan VB, et al: Disseminated tuberculosis masquerading as metastatic breast carcinoma on PET-CT. Clin Nucl Med 33: 359-361, 2008.

31. Li YJ, Cai L, Sun HR, et al: Increased FDG uptake in bilateral adrenal tuberculosis appearing like malignancy. Clin Nucl Med 33: 191-192, 2008

32. Lin WY, Hung GU and Tsai SC: A pitfall of FDG-PET image interpretation: accumulation of FDG in the dependent area of the urinary bladder after bladder irrigation - the usefulness of the prone position. Clin Nucl Med 30: 638-639, 2005. 